The $T_{k}$ and $S_{k}$ tests given by Dickson, Townes, and Hall are derivable from these tests by consideration of the requirements imposed by test (d) on the $T_{k}$ and $S_{k}$.

Using a small linear congruence machine developed by $\mathrm{D}$. $\mathrm{H}$. Lehmer, and with the kind assistance of Prof. and Mrs. Lehmer, the author checked the possible discriminants to $10^{7}$, verifying the following theorem.

THEOREM: There are no discriminants with a single class in each genus, $3315<\Delta<10,000,000$.

The largest prime necessary in this test was 79.

California Institute of Technology

\title{
ON FINITE EXTENDING GROUPS
}

\section{ALBERT NEWHOUSE ${ }^{1}$}

In his paper Non-associative algebras, ${ }^{2}$ A. A. Albert defined extending groups $\mathbb{B}$ for algebras $\mathfrak{A}$ with a unity element. ${ }^{3}$ Such groups are merely finite multiplicative groups of nonsingular linear transformations on a linear space $\mathfrak{A}$ of order $n>1$ over a field $\mathfrak{F}$ defined so that all the transformations leave the unity element $e$ of $\mathfrak{A}$ unaltered. With respect to the basis $\left(e, u_{2}, u_{3}, \cdots, u_{n}\right)$ of $\mathfrak{A}$ over $\mathfrak{F}$ these groups are then isomorphic to finite groups $(B)$ of $n$-rowed square matrices of the form

$$
G=\left(\begin{array}{ll}
1 & 0 \\
B & M
\end{array}\right)
$$

where $M$ is an $(n-1)$-rowed nonsingular square matrix and $B$ a 1 by $n-1$ matrix.

In his paper Albert ${ }^{4}$ has raised the question of the existence of such groups (\&) "such that no basis of $\mathfrak{A}$ exists for which \& may be regarded as a permutation group."

Presented to the Society, April 29, 1944; received by the editors March 14, 1944, and, in revised form, April 18, 1947, and July 29, 1947.

1 The author is indebted to the referee for his helpful comments.

${ }^{2}$ Ann. of Math. vol. 43 (1942) pp. 685-723.

8 Ibid. p. 712.

4 Ibid. Footnote, p. 722. 
We shall prove that such groups exist for every algebra $\mathfrak{A}$ whose order $n>2$ over $\mathfrak{F}$ and shall completely settle the case $n=2$.

If $n>2$ and the characteristic of $\mathfrak{F}$ is different from 2 then the matrix

$$
G=\left(\begin{array}{crr}
I_{n-2} & 0 & 0 \\
0 & -1 & 0 \\
0 & 0 & -1
\end{array}\right)
$$

where $I_{n-2}$ is the identity matrix of order $n-2$, generates a cyclic group (S) of order 2. The minimum function of $G$ is $x^{2}-1$, its characteristic function is $(x-1)^{n-2}(x+1)^{2}$. This group is isomorphic to the permutation group of order $2, \mathfrak{B}=\left[I_{n}, P\right]$, with $P$ similar to

$$
\left(\begin{array}{ccc}
I_{n-2} & 0 & 0 \\
0 & 0 & 1 \\
0 & 1 & 0
\end{array}\right) .
$$

The minimum function of $P$ is $x^{2}-1$, its characteristic function is $(x-1)^{n-2}\left(x^{2}-1\right)=(x-1)^{n-1}(x+1)$. Thus $G$ is not similar to $P$ and $\&$ is not a permutation group on any base of $\mathfrak{A}$.

Now let the characteristic of $\mathfrak{F}$ be 2 . If $n$ is not a power of 2 then there exists an integer $m$ such that $2^{m}>n>2^{m-1}$. Let $M_{m}$ be the companion matrix of $x^{2^{m-1}}+1$, a square matrix of $2^{m-1}$ rows. Now let

$$
N_{m}=\left(\begin{array}{lll}
1 & 0 & 0 \cdots 0 \\
1 & & \\
0 & & M_{m} \\
\vdots & & \\
0 & &
\end{array}\right),
$$

a square matrix of $2^{m-1}+1$ rows.

Then

$$
G=\left(\begin{array}{cc}
I_{n-2^{m-1}-1} & 0 \\
0 & N_{m}
\end{array}\right)
$$

will generate a cyclic group of order $2^{m}$ since the characteristic and minimum function of $N_{m}$ is $(x+1)^{2^{m-1}+1}$, a divisor of $(x+1)^{2^{m}}$ $=x^{2^{m}}+1$ and not a divisor of $(x+1)^{2^{m-1}}=x^{2^{m-1}}+1$. Thus $G$ is of order $2^{m}$ and $G$ cannot be any permutation of $n$ letters since one cycle would have to have $2^{m}>n$ letters.

If $n=2^{m}>4$, let 


$$
N=\left(\begin{array}{ll}
0 & 1 \\
1 & 1
\end{array}\right)
$$

whose characteristic and minimum function is $x^{2}+x+1$. Then let

$$
G=\left(\begin{array}{ccc}
I_{2^{m-1}-3} & 0 & 0 \\
0 & N_{m} & 0 \\
0 & 0 & N
\end{array}\right),
$$

an $n$-rowed square matrix. Its characteristic function is $(x+1)^{n-2}$ $\cdot\left(x^{2}+x+1\right)$, its minimum function is $(x+1)^{2^{m-1}+1}\left(x^{2}+x+1\right)$ which is a divisor of $x^{3.2^{m}}+1=\left(x^{3}+1\right)^{2^{m}}=(x+1)^{2^{m}}\left(x^{2}+x+1\right)^{2^{m}}$. Thus $G$ is of order $3 \cdot 2^{m}$. No permutation on $n=2^{m}$ letters is of order $3 \cdot 2^{m}$ since one cycle would have to have 3 letters and one cycle $2^{m}$ letters.

If $n=4$ let

$$
G=\left(\begin{array}{llll}
1 & 0 & 0 & 0 \\
1 & 1 & 0 & 0 \\
0 & 0 & 0 & 1 \\
0 & 0 & 1 & 1
\end{array}\right) .
$$

Its characteristic and minimum function is $x^{4}+x^{3}+x+1$, a divisor of $x^{6}+1=\left(x^{4}+x^{3}+x+1\right)\left(x^{2}+x+1\right)$. Thus $G$ is of order 6 and not similar to a permutation matrix since the corresponding permutation matrix would have to have cycles of 3 and 2 letters each, and there are only 4 letters.

If $n=2$ the extending group $\$$ consists of 2 -rowed square matrices

$$
G=\left(\begin{array}{ll}
1 & 0 \\
a & b
\end{array}\right)
$$

Let $m$ be the order of $\$$, then $G^{m}=I_{2}$, but

$$
G^{m}=\left(\begin{array}{cc}
1 & 0 \\
a\left(1+b+\cdots+b^{m-1}\right) & b^{m}
\end{array}\right),
$$

thus $b^{m}=1$ and $b$ is an $m$ th root of unity. Thus if $\mathfrak{F}$ contains a primitive $m$ th root of unity $b$ for $m>2$ then

$$
G=\left(\begin{array}{ll}
1 & 0 \\
0 & b
\end{array}\right)
$$

generates a cyclic group of order $m$. The characteristic function of $G$ is $x^{2}-(b+1) x+b$, different from the characteristic function of any 
permutation matrix on two letters.

If $\mathfrak{F}$ does not contain any roots of unity besides 1 and $-1, b$ must be 1 or -1 and $G$ has the form

$$
G_{1}=\left(\begin{array}{ll}
1 & 0 \\
a & 1
\end{array}\right) \text { or } G_{2}=\left(\begin{array}{cc}
1 & 0 \\
a & -1
\end{array}\right)
$$

so that

$$
G_{1}^{m}=\left(\begin{array}{cc}
1 & 0 \\
m a & 1
\end{array}\right), \quad G_{2}^{2}=\left(\begin{array}{ll}
1 & 0 \\
0 & 1
\end{array}\right)=I .
$$

If $\mathfrak{F}$ is non-modular $\$$ can only contain elements of form $G_{2}$. Now let

$$
S=\left(\begin{array}{cc}
1 & 0 \\
a & -1
\end{array}\right), \quad T=\left(\begin{array}{cc}
1 & 0 \\
b & -1
\end{array}\right), \quad a \neq b, \quad S^{2}=T^{2}=I,
$$

then

$$
S T=\left(\begin{array}{cc}
1 & 0 \\
a-b & 1
\end{array}\right)
$$

$S T$ is of form $G_{1}$ and cannot be in $\$$. Thus for $n=2$ and $\mathfrak{F}$ nonmodular there exist finite extending groups only if $\mathfrak{F}$ contains a primitive $m$ th root of unity for $m>2$.

If $\mathfrak{F}$ is of characteristic $p>2$,

$$
G=\left(\begin{array}{ll}
1 & 0 \\
1 & 1
\end{array}\right)
$$

generates a cyclic group of order $p$. This group is not a permutation group on two letters since such a group has order two.

If $\mathfrak{F}$ is of characteristic 2 and contains an extension of the prime field $G F(2)$ then $\mathfrak{F}$ contains at least four elements $0,1, a, 1+a$, $(a \neq 0,1)$. Then

$$
\begin{gathered}
I, \quad R=\left(\begin{array}{ll}
1 & 0 \\
a & 1
\end{array}\right), \quad S=\left(\begin{array}{cc}
1 & 0 \\
1+a & 1
\end{array}\right), \\
R S=S R=\left(\begin{array}{ll}
1 & 0 \\
1 & 1
\end{array}\right), \quad R^{2}=S^{2}=(R S)^{2}=(S R)^{2}=I,
\end{gathered}
$$

form a group of order 4 not a permutation group on two letters, since two letters have only two permutations.

If $\mathfrak{F}=G F(2)$, the only nonsingular linear transformations of the prescribed form are 


$$
I=\left(\begin{array}{ll}
1 & 0 \\
0 & 1
\end{array}\right), \quad G=\left(\begin{array}{ll}
1 & 0 \\
1 & 1
\end{array}\right) .
$$

However, the characteristic and minimum function of $G$ is $(x+1)^{2}$ $=x^{2}+1$ and $G$ is similar to the permutation matrix

$$
P=\left(\begin{array}{ll}
0 & 1 \\
1 & 0
\end{array}\right)
$$

Thus we have the following theorem.

THEOREM. For every finite algebra $\mathfrak{A}$ over $\mathfrak{F}$ there exist finite extending groups $\&$ which are not permutation groups on any basis of $\mathfrak{A}$ if the order of $\mathfrak{A}$ over $\mathfrak{F}$ is greater than 2.

If the order of $\mathfrak{A}$ over $\mathfrak{F}$ is 2 there exist such extending groups if and only if

(a) $\mathfrak{F}$ is non-modular and contains a primitive mth root of unity for $m>2$,

(b) $\mathfrak{F}$ is of characteristic $p$ and contains more than two elements.

UNIVERSITY OF HoUstoN 